\title{
Melting line of charged colloids from primitive model simulations
}

\author{
Antti-Pekka Hynninen ${ }^{\text {a) }}$ and Marjolein Dijkstra \\ Soft Condensed Matter Group, Debye Institute, Utrecht University, Princetonplein 5, 3584 CC Utrecht, \\ The Netherlands
}

(Received 28 June 2005; accepted 24 October 2005; published online 28 December 2005)

\begin{abstract}
We develop an efficient simulation method to study suspensions of charged spherical colloids using the primitive model. In this model, the colloids and the co- and counterions are represented by charged hard spheres, whereas the solvent is treated as a dielectric continuum. In order to speed up the simulations, we restrict the positions of the particles to a cubic lattice, which allows precalculation of the Coulombic interactions at the beginning of the simulation. Moreover, we use multiparticle cluster moves that make the Monte Carlo sampling more efficient. The simulations are performed in the semigrand canonical ensemble, where the chemical potential of the salt is fixed. Employing our method, we study a system consisting of colloids carrying a charge of 80 elementary charges and monovalent co- and counterions. At the colloid densities of our interest, we show that lattice effects are negligible for sufficiently fine lattices. We determine the fluid-solid melting line in a packing fraction $\eta$-inverse screening length $\kappa$ plane and compare it with the melting line of charged colloids predicted by the Yukawa potential of the Derjaguin-Landau-Verwey-Overbeek theory. We find qualitative agreement with the Yukawa results, and we do not find any effects of many-body interactions. We discuss the difficulties involved in the mapping between the primitive model and the Yukawa model at high colloid packing fractions $(\eta>0.2)$. ( 2005 American Institute of Physics. [DOI: 10.1063/1.2138693]
\end{abstract}

\section{INTRODUCTION}

Charge-stabilized colloidal suspensions are mixtures of mesoscopic charged particles (e.g., latex spheres) suspended in a solvent (e.g., water or ethanol) with co- and counterions. Such suspensions are typical examples of systems with high charge and size asymmetries. Colloids can carry a charge up to 10000 elementary charges and their sizes are in the range of $1 \mu \mathrm{m}$, while the microions are typically monovalent with sizes of $\sim 4 \AA$. Direct simulations of charged colloids are often hampered by CPU time requirements. This is because high charge asymmetry requires a large number of counterions to counterbalance the colloid charge and high size asymmetry requires a small time step in molecular dynamics or a small trial displacement in Monte Carlo (MC) simulations. Therefore, most simulations of charged colloids are performed using coarse-grained models, in which the degrees of freedom of the microions are integrated out and the colloids interact with an effective (usually pairwise) potential. The most well-established coarse-grained model is based on the Derjaguin-Landau-Verwey-Overbeek (DLVO) theory. ${ }^{1,2}$ According to the DLVO theory, the counterions form a double layer of opposite charge around each colloid. The extent of the double layer depends on the concentration of added salt (co- and counterions). At low salt concentrations, the double layers are inflated, while at high salt concentrations, the double layers are thin. The coarse-grained entities, colloids with their double layers, interact via a hard-core repulsion due to the finite size of the colloids and a screened Coulomb (repulsive Yukawa) interaction, with the screening length

${ }^{a)}$ Electronic mail: a.p.hynninen@phys.uu.nl given by the thickness of the double layer. The phase diagram of hard-core repulsive Yukawa particles ${ }^{3,4}$ explains the experimentally observed phase behavior, ${ }^{5-8}$ where the colloids exhibit fluid, body-centered-cubic (bcc), and facecentered-cubic (fcc) phases.

In recent years, there has been a lot of debate whether the DLVO pairwise description is valid at low salt concentrations, where double layers of nearby colloids overlap. The debate was initiated by a variety of unexplained phenomena that have been observed in experiments of charge-stabilized colloidal suspensions at low salt concentrations, e.g., vaporliquid condensation, ${ }^{9}$ large stable "voids," lously long-lived dense clusters. ${ }^{12}$ A long-range attraction would account naturally for these phenomena, but is inconsistent with the long-accepted repulsive DLVO potential. Another explanation might be that the effective pair potential description fails at low salt concentrations and that the threeand higher-body interactions are important. This discussion was fueled by theoretical results of attractive three-body interactions $^{13,14}$ and experiments that show densitydependent pair interactions ${ }^{15,16}$ (signaling that the underlying interaction is not pairwise). By now, the existence of manybody interactions has been established experiment and numerical calculations. ${ }^{17-19}$

What remains to be less clear-cut is the effect of the many-body interactions on the phase behavior of charged colloids. Dobnikar et al. ${ }^{20-22}$ have studied the melting transition by solving the nonlinear Poisson-Boltzmann equation for a multicolloid system. They found that the effective many-body interactions shift the melting line, extending the fluid range, than expected on the basis of pairwise interactions alone. In our earlier work, ${ }^{23,24}$ we studied the effect of 
attractive three-body interactions on the phase behavior of charged colloids and found that they give rise to broad fluidfcc and bcc-fcc coexistence regions. The question remains whether the effective three-body approach, which ignores the effective four- and higher-body terms, is valid at high colloid densities. This question becomes even more important in the light of recent experimental evidence, which suggests that the four-body term is repulsive ${ }^{19}$ and of the same range and magnitude as the attractive three-body interaction. Hence, a substantial fraction of the three-body attraction seems to be canceled by the four-body repulsion.

In order to resolve this issue, we decided to perform direct simulations of the primitive model, using the same parameters as in our earlier work, where triplet interactions are important. $^{23,24}$ To this end, we develop an efficient simulation method for the highly asymmetric primitive model. In the primitive model description, colloids, coions, and counterions are treated as charged hard spheres, while the solvent is treated as a structureless continuum characterized by a dielectric constant $\epsilon_{s}$. Since the primitive model includes all the charged species explicitly, it also includes all the effective many-body interactions. Employing our simulation method, we determine the fluid-solid transition at various salt concentrations for the primitive model and we compare the results with the predictions of the DLVO theory.

In comparing the primitive model results with the effective Yukawa potential of the DLVO theory, it is important to know how to map the primitive model parameters onto the Yukawa model parameters. At low colloid packing fractions, this mapping can be done quite reliably using the PoissonBoltzmann cell model, ${ }^{25}$ see, e.g., Refs. 20 and 26. At high colloid packing fractions $\eta$, less is known about the validity of the Yukawa potential ${ }^{27}$ and the way the parameters should be mapped. An example of this can be found in Refs. 28-30, where highly charged colloidal suspensions were studied using a Car-Parrinello-type $a b$ initio method. ${ }^{31}$ At high $\eta$, it was found that the DLVO theory underestimates the structure, meaning that the colloids see an effective charge that is higher than the real colloidal charge. Supposedly, the reason for this is that at high $\eta$, the screening of the Coulomb interactions is reduced because the counterions are excluded by the neighboring colloids. ${ }^{30}$ We find evidence of this charge renormalization, but we are not able to directly prove the mechanism behind it. We are unable to find a mapping between the primitive model and the Yukawa model that would work at all packing fractions.

The paper is organized as follows. In Sec. II we present our simulation method for the highly asymmetric primitive model. In Sec. III, we discuss our results and we end with some concluding remarks in Sec. IV.

\section{SIMULATION METHOD}

\section{A. Highly asymmetric primitive model}

Our system consists of $N$ colloidal hard spheres with a diameter $\sigma$, carrying a positive charge $+Z e$, and $N_{S}$ co- and $Z N+N_{S}$ counterions with a diameter $\sigma_{m}$ and a charge $+e$ and $-e$, respectively. Here $e$ denotes the elementary charge. The particles interact via the pairwise additive Coulombic potential and a hard-core repulsion

$$
\frac{U_{i j}(r)}{k_{B} T}=\left\{\begin{array}{cc}
\frac{q_{i} q_{j} \lambda_{B}}{\sigma} \frac{\sigma}{r} & \text { for } r \geqslant \frac{1}{2}\left(\sigma_{i}+\sigma_{j}\right) \\
+\infty & \text { for } r<\frac{1}{2}\left(\sigma_{i}+\sigma_{j}\right),
\end{array}\right.
$$

where $\lambda_{B}=e^{2} / \epsilon_{s} k_{B} T$ is the Bjerrum length, $\epsilon_{s}$ is the dielectric constant of the solvent, and $r$ is the distance between the ions $i$ and $j$ that have charge numbers $q_{i}$ and $q_{j}$.

Our primitive model Monte Carlo simulations are performed in the semigrand canonical ensemble, i.e., the volume $V$, temperature $T$, number of colloids $N$, and chemical potential of the salt $\mu_{S}$ are kept constant. The simulations represent a system of colloids in osmotic equilibrium with a salt reservoir at the same chemical potential $\mu_{S}$. In our simulations, one $\mathrm{MC}$ cycle consist of $N(Z+1)+2 N_{S}$ attempts to displace a randomly selected particle (a colloid or a microion). Attempts to insert or remove a coion-counterion pair are performed ten times per MC cycle. We use the standard grand canonical ensemble method for the insertion and removal steps. ${ }^{32}$

The simulations are performed in a cubic box with side length $L$ and periodic boundary conditions are applied in all three dimensions. The long-range Coulombic interactions are calculated using the Ewald summation method ${ }^{32,33}$ with conducting boundary conditions, 518 Fourier-space vectors, and real-space damping parameter $\alpha=5$. In order to speed up the simulations, we have used the fine lattice discretization method of Panagiotopoulos and Kumar. ${ }^{34}$ In this method, the positions of the particles are restricted to a cubic lattice with lattice spacing $a$. We introduce a lattice refinement parameter $\xi=\sigma / a$, which gives the number of lattice points per colloid diameter. Continuum is recovered in the limit of $\xi \rightarrow \infty$. Previously, the lattice method has been used to study the criticality of electrolytes ${ }^{34-38}$ and polyelectrolytes. ${ }^{39}$ In these systems, the lattice has little impact on the critical behavior once $\xi \geqslant 3 .{ }^{34}$ The effect of lattice discretization on hard spheres has been studied in Ref. 40. The advantage of using a lattice is that it allows a single precalculation of all the Coulombic interactions into a three-dimensional $(L / a) \times(L / a) \times(L / a)$ matrix at the beginning of the simulation. During a simulation, the pair interactions can be determined by a simple table lookup. Figure 1 shows the CPU time $\tau$ as a function of the total number of charges $N_{\text {tot }}=N(Z+1)+2 N_{S}$ of both the Ewald summation method and the lattice method and the inset shows the ratio between the two CPU times. ${ }^{41}$ The Ewald parameters were chosen as described in Ref. 32. The full lines in Fig. 1 are linear fits to the data points. From these fits we see that the CPU time of the Ewald summation method scales as $N_{\text {tot }}^{1.73}$ and the CPU time of the lattice method scales as $N_{\text {tot }}^{2.03}$. We note that the lattice method has the expected scaling, while the Ewald summation does not have the ideal $N_{\text {tot }}^{1.5}$ scaling. ${ }^{42}$ In the Ewald summation, a slightly nonideal scaling is obtained because we do not use neighbor lists in the total potential-energy calculation, and therefore, the distance between each particle has to be calculated. We are not sure how much better the Ewald summation 


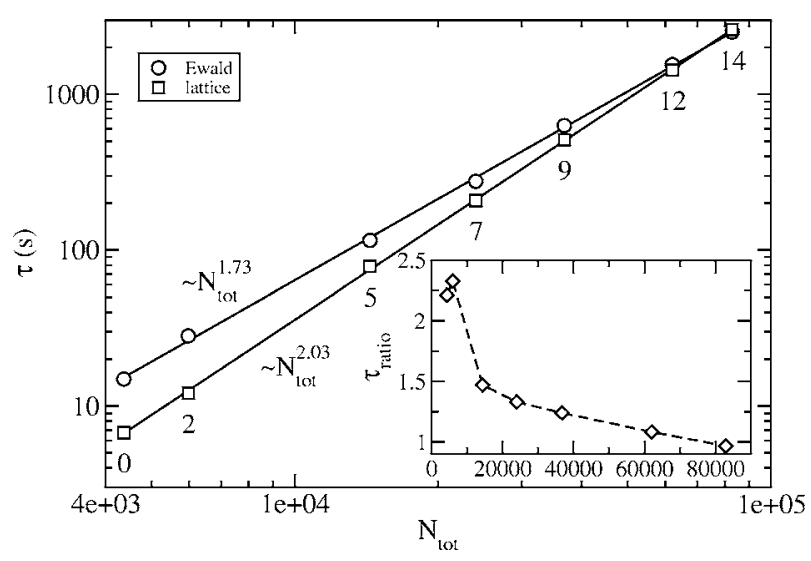

FIG. 1. CPU time $\tau$ vs the total number of charge centers $N_{\text {tot }}=N(Z+1)$ $+2 N_{S}$ for a system with $Z=80, \eta=0.2$, and $\xi=19$. The circles $(\bigcirc)$ mark the results of the Ewald summation and the squares $(\square)$ mark the results of the lattice method. The annotated numbers show the $\kappa_{\mathrm{id}} \sigma$ values. The full lines are linear fits to the data points. The inset shows the ratio of the Ewald summation and the lattice method CPU times.

would perform if neighbor lists were used since the construction and updating of the lists also require CPU time.

In a system with a high density of microions, a simple displacement of a colloid would almost always result in an overlap with at least one of the microions. In order to overcome this problem, we remove the microions that hinder the displacement of the colloid at its new position and reinsert them into the space vacated by the displacement of the colloid. Figure 2(a) shows a typical starting configuration. The colloid at its new trial position is denoted by a dashed line and the microions that result in an overlap with the colloid at its new position are filled gray. In our cluster move, the overlapping microions are reflected through the center between the old and new colloid positions (marked P) into the space left empty by the displacement of the colloid. An example of such a reflection is shown in Fig. 2(a) by the gray arrow. Figure 2(b) shows the end situation, where the colloid and the microions are at their new positions. Cluster moves are performed only in three lattice coordinate directions, which guarantee that each reflected microion has a well-defined lattice position. The cluster moves satisfy detailed balance as they are entirely symmetric, i.e., moving a colloid back to its original position returns the microions to their original positions. Our method is similar to the cluster move technique used in Ref. 43. However, in their approach, also the counterions close to the colloids are added in the cluster move. In our case, these extended cluster moves are not needed as the coupling between the colloid and counterions is weak.

We define the (inverse) Debye screening length of the reservoir as

$$
\kappa \sigma=\sqrt{8 \pi \lambda_{B} \sigma^{2}\left\langle\rho_{S}^{\text {res }}\right\rangle},
$$

where $\left\langle\rho_{S}^{\text {res }}\right\rangle=\left\langle N_{S}\right\rangle / V$ is the average salt density in the reservoir measured from a simulation with no colloids $(N=0)$ and a fixed $\mu_{S}$. In the ideal-gas approximation, the salt chemical potential $\mu_{S}$ is related to the (ideal) salt reservoir density $\rho_{S}^{\text {res }}$ through
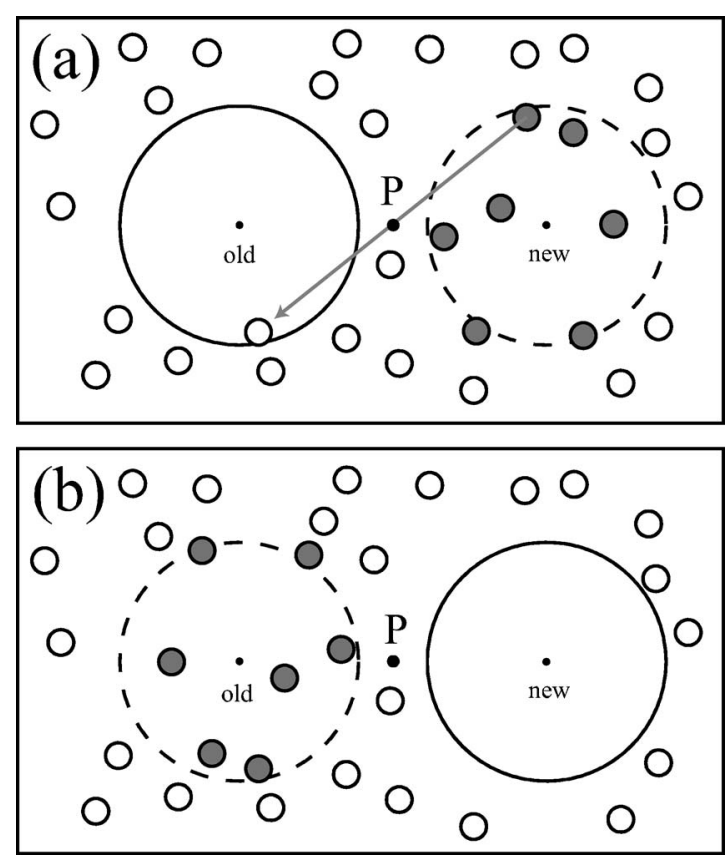

FIG. 2. The multiparticle cluster move technique. In (a), the colloid is at its old position and the colloid at its new trial position is drawn with a dashed line. The gray microions overlap with the colloid at its new position and are therefore included in the cluster move. The reflection point $\mathrm{P}$ is in the middle between the old and new colloid positions. The gray arrow shows an example of a reflection through point $\mathrm{P}$. In (b), the colloid is at its new position and the microions (gray) are reflected to their new positions.

$$
\mu_{S}=2 k_{B} T \ln \left(\rho_{S}^{\mathrm{res}} \sigma^{3}\right),
$$

and one can define an ideal reservoir screening length as

$$
\kappa_{\mathrm{id}} \sigma=\sqrt{8 \pi \lambda_{B} \sigma^{2} \rho_{S}^{\text {res }}} .
$$

We tested the validity of the ideal-gas approximation in Eq. (3) by performing simulations of a reservoir system $(N=0)$. The simulations were performed in a box with side length $L=2.11 \sigma$, Bjerrum length $\lambda_{B}=0.0225 \sigma$, microion diameter $\sigma_{m}=\sigma / 19$, and lattice parameter $\xi=38$. For a fixed $\mu_{S}$, we measured $\left\langle\rho_{S}^{\text {res }}\right\rangle$ in a simulation and calculated the reservoir screening length $\kappa \sigma$ using Eq. (2). In Fig. 3, we plot the reservoir screening length $\kappa \sigma$ as a function of the ideal reservoir screening length $\kappa_{\mathrm{id}} \sigma$. The dashed line gives the idealgas result, where $\kappa \sigma=\kappa_{\mathrm{id}} \sigma$. As can be seen from Fig. 3, the reservoir screening length $\kappa \sigma$ starts to deviate from the ideal reservoir screening length at $\kappa_{\mathrm{id}} \sigma>6$, but the difference remains small even up to $\kappa_{\mathrm{id}} \sigma=10$, where $\kappa \sigma \approx 9.5$. Therefore, Eqs. (3) and (4) give a convenient way of approximating $\kappa \sigma$ at a given salt chemical potential $\mu_{S}$.

In summary, we present an efficient simulation method to study model suspensions of charged colloids using the primitive model. Primitive model simulations cause severe sampling problems as the number of particles involved in the simulations increases with larger charge asymmetry and the convergence slows down due to the high density of microions close to the colloids. The combination of the fine lattice discretization approach and the cluster moves enables us to solve some of the sampling problems. We estimate that the lattice gives a speed up of a factor 2 compared with continuum simulations in the range of parameters where most of 


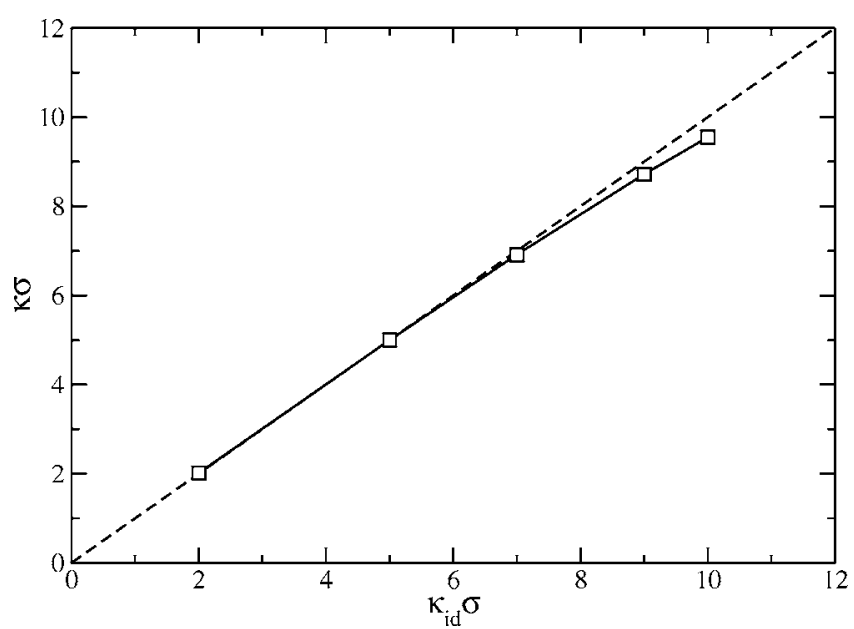

FIG. 3. The reservoir screening length $\kappa \sigma$ as a function of the ideal reservoir screening length $\kappa_{\mathrm{id}} \sigma$. The dashed line gives the ideal-gas result, where $\kappa \sigma=\kappa_{\mathrm{id}} \sigma$. The error bars are not shown as they are smaller than the symbol sizes. The solid line is a guide to the eye.

our simulations were performed, and the cluster move technique gives a speed up of 10 or more. Therefore, the cluster move technique is considered essential to make the simulations feasible. These methods are a combination of methods used previously. For previous studies on highly charged colloidal suspensions, see, e.g., Refs. 43-45. See also a recent review ${ }^{46}$ and the references therein. Distinct from previous work, our simulations are performed in the semigrand canonical ensemble, i.e., the salt chemical potential and, hence, the Debye screening length of the reservoir are fixed. This facilitates a direct comparison with theoretical predictions. To the best of our knowledge, we are not aware of any simulation study of the primitive model at fixed salt chemical potential in the colloidal regime.

\section{B. Hard-core repulsive Yukawa model}

In addition to the primitive model simulations, we also perform MC simulations of the hard-core Yukawa model. According to the DLVO theory, ${ }^{1,2}$ the colloid-colloid pair interaction is given by a repulsive Yukawa potential with a hard-core,

$$
\frac{u(r)}{k_{B} T}= \begin{cases}\frac{Z^{2} \lambda_{B} / \sigma}{(1+\kappa \sigma / 2)^{2}} \frac{\exp [-\kappa(r-\sigma)]}{r / \sigma} & \text { for } r \geqslant \sigma \\ +\infty & \text { for } r<\sigma .\end{cases}
$$

Simulations of the continuum hard-core Yukawa model are performed in the canonical ensemble using a cubic box with periodic boundary conditions. The DLVO theory is derived for infinite dilution where $\eta=0$. At $\eta>0$, it is customary to replace the reservoir screening length $\kappa$ in Eq. (5) by an effective screening length $\kappa_{\text {eff }}$. The effective screening length $\kappa_{\text {eff }}$ takes into account that, at a finite colloid density, the screening length in the colloidal suspensions is not simply determined by that of the reservoir (where the colloid density is zero). The reason is that the counterions from other colloids also contribute to the screening length. A simple way to incorporate this extra contribution is to substitute the reser- voir charge density $2\left\langle\rho_{S}^{\text {res }}\right\rangle$ in Eq. (2) with the average total microion charge density $2\left\langle\rho_{S}\right\rangle+Z \rho$

$$
\kappa_{\mathrm{eff}} \sigma=\sqrt{4 \pi \lambda_{B} \sigma^{2}\left(2\left\langle\rho_{S}\right\rangle+Z \rho\right)},
$$

where $\rho=N / V$ is the colloid number density and $\left\langle\rho_{S}\right\rangle$ $=\left\langle N_{S}\right\rangle / V$ is the average salt density measured from a primitive model simulation. We also consider an effective screening length where the excluded volume of the colloids is taken into account (see, e.g., Ref. 47),

$$
\kappa_{\mathrm{eff}}^{\prime} \sigma=\sqrt{4 \pi \lambda_{B} \sigma^{2}\left(\frac{2\left\langle\rho_{S}\right\rangle}{1-\eta_{c}}+\frac{Z \rho}{1-\eta_{c}}\right)},
$$

where $\eta_{c}=\eta\left(1+\sigma_{m} / \sigma\right)^{3}$ is the fraction of the volume excluded by the colloids. For highly charged colloids, it is typical to replace the bare charge $Z$ in Eq. (5)-(7) with a renormalized charge $Z_{\text {re }}$. The renormalized charge $Z_{\text {re }}$ takes into account the condensation of counterions on the colloid surface, see, e.g., Refs. 25 and 27. As $Z_{\text {re }}$ is a sum of the colloid charge $Z$ and the charge in the condensed shell of counterions, it is smaller than the bare charge $Z$. In the simulations presented in this paper, charge renormalization is insignificant (see also Sec. III C).

At low colloid concentrations, there are both numerical and experimental evidence that the Yukawa form (5) is valid at high coupling, ${ }^{44}$ high charge, ${ }^{26,48}$ and high salt concentrations. $^{20-22}$ At low salt concentrations, one observes a "cutoff" behavior: at large distances the force between a pair of colloids is considerably smaller than expected on the basis of the Yukawa potential. ${ }^{15,16,20-22,49}$ Hence, the effective colloid-colloid pair interaction resembles closely a Yukawa potential that has a density-dependent truncation. Phase diagrams of such potentials have been studied in Refs. 20-22 and 50.

As was mentioned in the Introduction, much less is known about the validity of the Yukawa form at high colloid concentrations, even with effective parameters (see, e.g., the discussion in Ref. 27). In Refs. 28-30, highly charged colloidal suspensions were studied using an $a b$ initio method that can be seen as a classical counterpart of the CarParrinello method. ${ }^{31}$ It was found that the Yukawa potential is valid at all packing fractions, but that the parameters of the potential do not follow the DLVO theory. At low $\eta$, the DLVO theory overestimates the structure, as expected according to the charge renormalization discussed above. At high $\eta$, the DLVO theory underestimates the structure, meaning that the colloids see an effective charge that is higher than the bare charge. The reason for this is that at high $\eta$, the screening of the Coulomb interactions is reduced because the counterions are excluded by the neighboring colloids. ${ }^{30}$ Therefore, the colloids experience a stronger repulsion than expected based on the DLVO theory. The effect of the excluded volume on the effective pair potential is included by Belloni $^{51}$ (see also Ref. 27) by imposing that the structure factor within an integral equation theory using the mean spherical approximation (MSA) is the same in the effective one-component system and the primitive model. According to Belloni, ${ }^{51}$ the effective charge $Z_{\text {eff }}$ can be calculated from 


$$
Z_{\mathrm{eff}}=Z X(1+\kappa \sigma / 2) \exp (-\kappa \sigma / 2),
$$

where

$$
X=\cosh \left(\frac{\kappa \sigma}{2}\right)+U\left[\frac{\kappa \sigma}{2} \cosh \left(\frac{\kappa \sigma}{2}\right)-\sinh \left(\frac{\kappa \sigma}{2}\right)\right]
$$

and

$$
U=\frac{3 \eta}{(1-\eta)(\kappa \sigma / 2)^{3}}-\frac{\Gamma \sigma}{\kappa \sigma[1+\Gamma \sigma / 2+3 \eta /(1-\eta)]},
$$

and where $\Gamma$ can be solved from

$$
(\Gamma \sigma)^{2}=(\kappa \sigma)^{2}+\frac{24 \eta \lambda_{B} / \sigma}{1+\Gamma \sigma / 2+3 \eta /(1-\eta)} .
$$

In practice, one solves Eq. (11) numerically for a given $\kappa \sigma, \eta$, and $\lambda_{B} / \sigma$, and then evaluates Eqs. (8)-(10). The effective charge $Z_{\text {eff }}$ is larger than $Z$ at high $\eta$ and equal to $Z$ at low $\eta$. Our simulations are in the range where $Z_{\text {eff }}>Z$. The MSA theory is known to fail at low densities ${ }^{51}$ (see Ref. 26 for experimental evidence). At low densities, we also find that the MSA theory gives a worse description of the interactions than the DLVO theory (see Sec. III B).

In this paper, we determine the melting lines of the primitive and hard-core Yukawa models using the Lindemann criterion, ${ }^{52}$ which states that a crystal is stable when the root-mean-square (rms) displacement $\sqrt{\left\langle u^{2}\right\rangle}$ of the colloids about their equilibrium lattice positions $\left\{\mathbf{r}_{0, i}\right\}$ is below $19 \%$ of the mean colloid distance $\rho^{-1 / 3}=\sigma(\pi / 6 \eta)^{1 / 3}$, i.e.,

$$
\sqrt{\left\langle u^{2}\right\rangle} \equiv \sqrt{\left\langle\left|\mathbf{r}_{i}-\mathbf{r}_{0, i}\right|^{2}\right\rangle}<0.19 \sigma\left(\frac{\pi}{6 \eta}\right)^{1 / 3} .
$$

We define the melting packing fraction $\eta_{\text {melt }}$ as the smallest $\eta$ for which Eq. (12) holds, i.e., where a crystal is still stable.

\section{RESULTS}

\section{A. Effect of lattice discretization}

Our system consists of colloids with charge $80 e$ and monovalent co- and counterions. We use Bjerrum length $\lambda_{B}$ $=0.0225 \sigma$ and microion diameter $\sigma_{m}=\sigma / 19 \approx 0.053 \sigma$. Typical simulation runs consist of 5000 equilibration cycles (attempts to displace each particle once) and 10000 production cycles. Statistical errors are estimated from ten block averages using the Student's $t$-test with 95\% confidence.

We study the effect of the lattice discretization on the average salt density $\left\langle\rho_{S}\right\rangle$, average total potential energy per particle $\langle U\rangle / N$, and rms displacement of the colloids $\sqrt{\left\langle u^{2}\right\rangle}$. The ensemble averages are calculated for lattice discretization parameters $\xi=19,38$, and 57 , and for two reservoir screening lengths, $\kappa \sigma=2$ and 5 . For $\kappa \sigma=2$, the simulations were started from a bcc crystal containing $N=54$ colloids; for $\kappa \sigma=5$, the simulations were started from a fcc crystal with $N=32$ colloids. Figures 4(a) and 4(b) show $\left\langle\rho_{S}\right\rangle$ as a function of the packing fraction $\eta$ for screening lengths $\kappa \sigma=2$ and 5, respectively. We clearly observe that $\xi=38$ and $\xi=57$ give results that are equivalent within the statistical accuracy, while $\xi=19$ overestimates $\left\langle\rho_{S}\right\rangle$. A similar conclusion can be drawn from the data for $\langle U\rangle / N$ shown in Figs. 5(a) and 5(b):
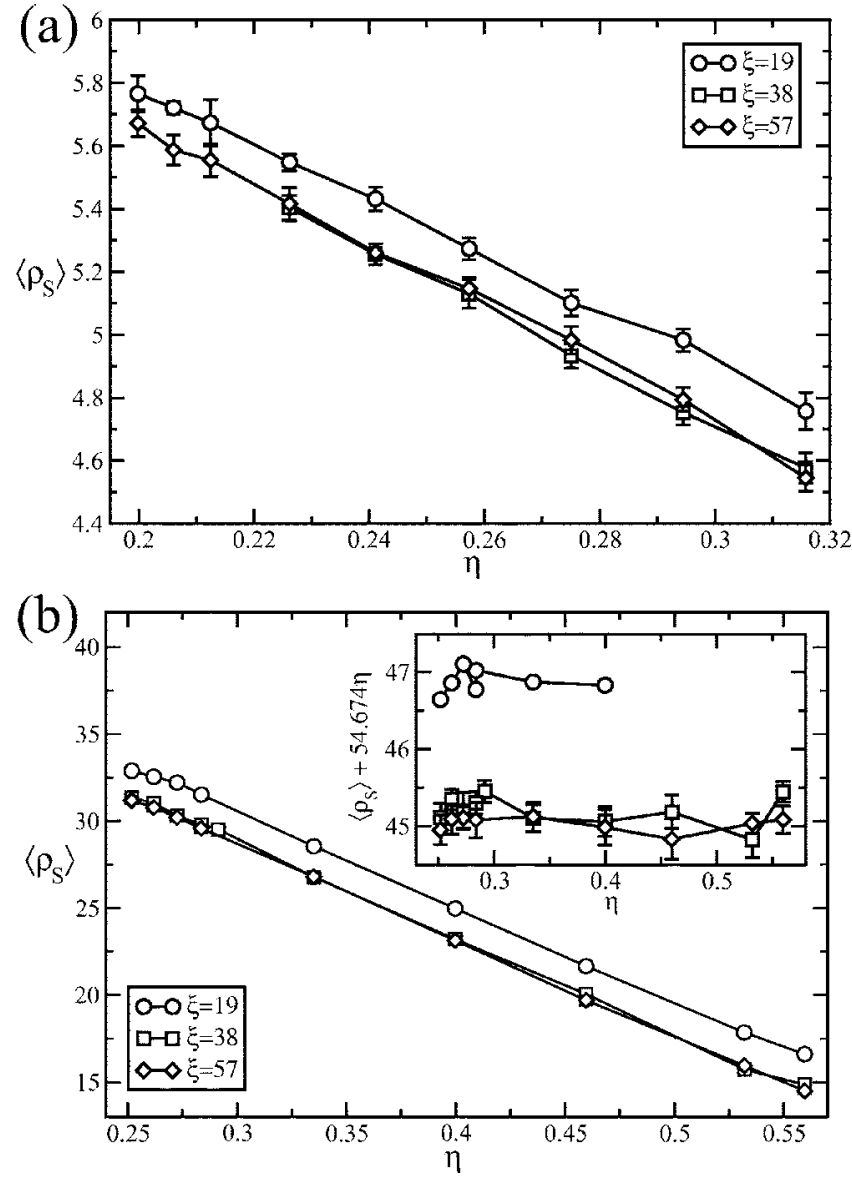

FIG. 4. The average salt density $\left\langle\rho_{S}\right\rangle$ (in units of $\sigma^{-3}$ ) as a function of the packing fraction $\eta$ for lattice parameters $\xi=19,38$, and $57 .\left\langle\rho_{S}\right\rangle$ in (a) is for a bcc crystal with $N=54$ colloids and reservoir screening length $\kappa \sigma=2 .\left\langle\rho_{S}\right\rangle$ in (b) is for a fcc crystal with $N=32$ colloids and $\kappa \sigma=5$. In the inset in (b), we subtract a linear fit from $\left\langle\rho_{S}\right\rangle$ to make the differences more visible. The lines are guides to the eye.

the results or the lattice discretization $\xi=38$ and $\xi=57$ are again equivalent within the statistical accuracy, while those for $\xi=19$ differ systematically. Figures 6(a) and 6(b) show the rms displacement in units of the mean colloid distance, $\sqrt{\left\langle u^{2}\right\rangle} / \rho^{-1 / 3}$, for $\kappa \sigma=2$ and $\kappa \sigma=5$, respectively. For $\kappa \sigma=2$, in Fig. 6(a), the effect of the lattice discretization on $\sqrt{\left\langle u^{2}\right\rangle} / \rho^{-1 / 3}$ is not clear due to the statistical noise. For $\kappa \sigma$ $=5$, in Fig. 6(b), we clearly see that $\xi=19$ overestimates $\sqrt{\left\langle u^{2}\right\rangle} / \rho^{-1 / 3}$ compared with the results obtained for $\xi=38$ and 57. Again, we observe that the results for $\xi=38$ and 57 are equivalent within the statistical accuracy. To conclude, the lattice discretization $\xi=38$ is sufficient to yield results that are independent of $\xi$ and are similar to the continuum case. We will use $\xi=38$ (or in some cases $\xi=57$ ) in all subsequent calculations.

\section{B. Charge distribution and structure}

We study the distribution of co- and counterions in a bcc crystal with $N=54$ colloids at packing fraction $\eta=0.34$ and reservoir screening length $\kappa \sigma=5$. A snapshot of this system is shown in Fig. 7. Since we are interested in the ion distribution in a crystal of colloids, we fix the colloid positions and do MC moves only for the ions. Co- and counterion 


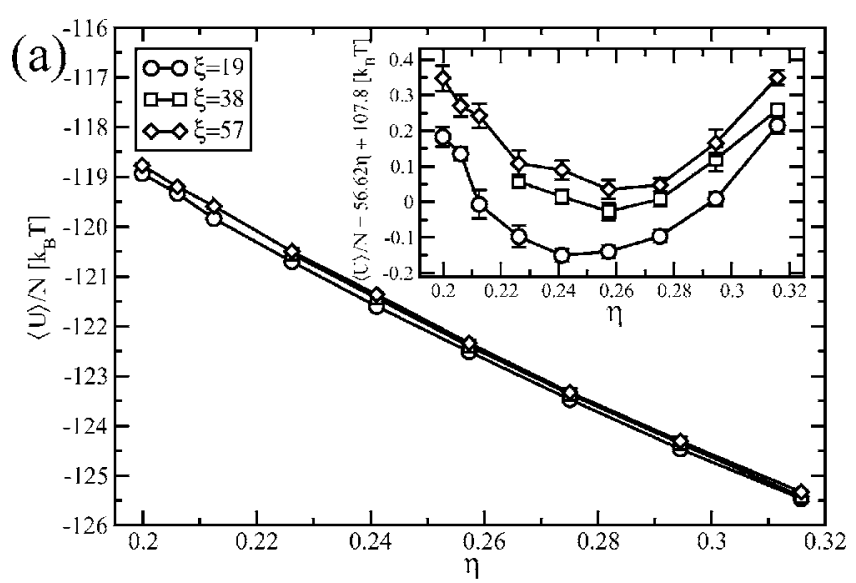

(b)

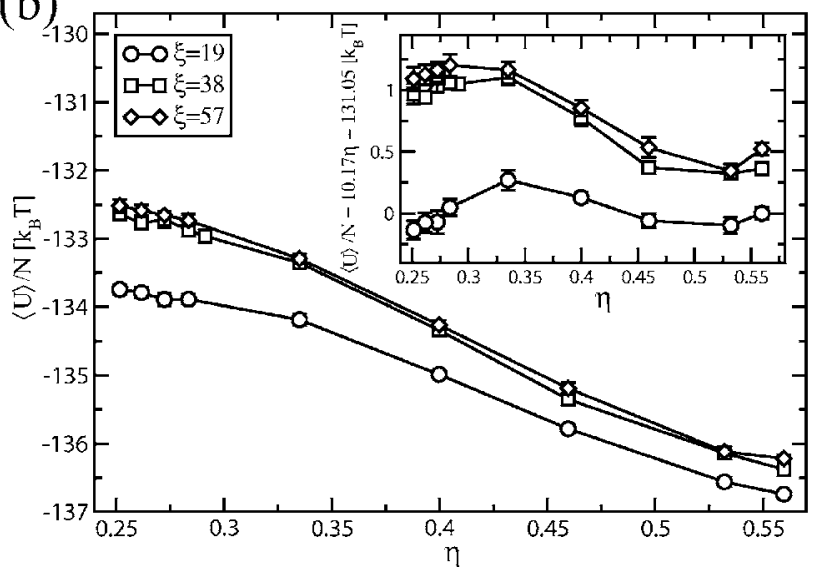

FIG. 5. The average potential energy per particle $\langle U\rangle / N$ (in units of $k_{B} T$ ) as a function of the packing fraction $\eta$ for lattice parameters $\xi=19,38$, and 57 . $\langle U\rangle / N$ in (a) is for a bcc crystal with $N=54$ colloids and reservoir screening length $\kappa \sigma=2 .\langle U\rangle / N$ in (b) is for a fcc crystal with $N=32$ colloids and $\kappa \sigma$ $=5$. In the insets, we subtract a linear fit from $\langle U\rangle / N$ to make the differences more visible. The lines are guides to the eye.

positions were recorded into 400 frames during $2000 \mathrm{MC}$ cycles. From this data, charge distributions were obtained by averaging the co- and counterion positions on a bcc unit cell that contains one colloid in the center and eight in the corners of a cube. The averages were saved on $60 \times 60 \times 60$ matrices, which were visualized by plotting the threedimensional (3D) constant-density contours with the GOPENMOL program. ${ }^{53}$ Figures 8 (a)-8(d) show 3D contour plots for [(a) and (b)] counterion, (c) coion, and (d) charge densities (counterion density minus coion density) in the bcc unit cell. Note that in Figs. 8(a), 8(c), and 8(d), the central colloid of the bcc unit cell is covered by the contour plots. The counterion contour plot in Fig. 8(a) shows that the counterions are distributed in a spherical orbital around each colloid, just like the DLVO theory predicts. Figure 8(b) shows a contour plot of counterions, where the constant-density contour is set at a higher counterion density than in Fig. 8(a). As can be seen from Fig. 8(b), the highest counterion density is on the part of the colloid surface where the nearest neighbors are closest to each other (i.e., between the center colloid and one of the corner colloids). This can be explained by superimposing two spherical orbitals, as is expected based on the DLVO theory. The coion contour plot in Fig. 8(c) shows that the coions are distributed in the regions left empty by the
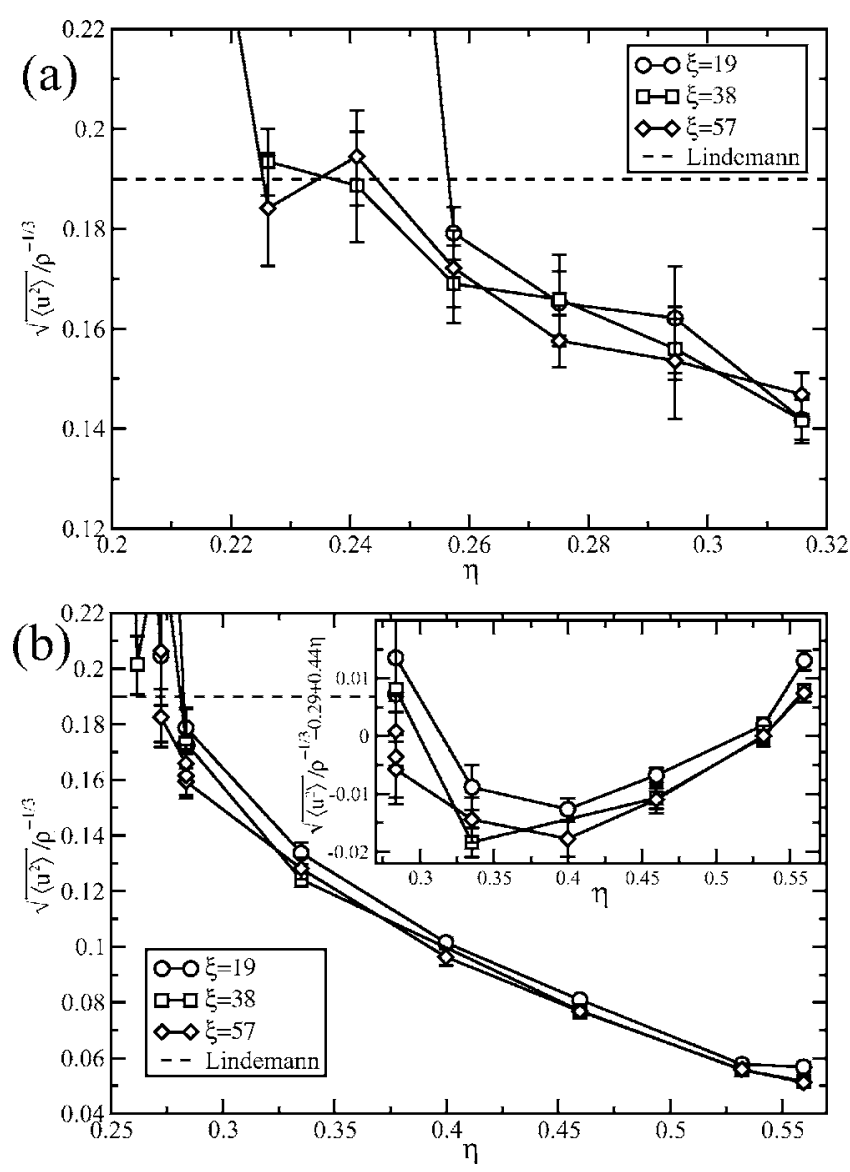

FIG. 6. The root-mean-square (rms) displacement $\sqrt{\left\langle u^{2}\right\rangle}$ in units of the mean colloid distance $\rho^{-1 / 3}$ as a function of packing $\eta$ for lattice parameters $\xi$ $=19,38$, and 57. The rms displacement in (a) is for a bcc crystal with $N$ $=54$ colloids and reservoir screening length $\kappa \sigma=2$. The rms displacement in (b) is for a fcc crystal with $N=32$ colloids and $\kappa \sigma=5$. The dashed line gives the Lindemann criterion. In the inset in (b), we subtract a linear fit from $\sqrt{\left\langle u^{2}\right\rangle} / \rho^{-1 / 3}$ to make the differences more visible. The lines are guides to the eye.

colloids and are depleted from the surfaces of the colloids. The highest density of coions is in the space between nextnearest neighbors (i.e., between the colloids in the corners of the unit cell). This region of space is still completely charge neutral, because it contains as many counterions as coions. This can be seen from Fig. 8(d), which shows a contour plot of the charge density (counterion density minus coion density). Figure 8(d) also shows that the charge distribution is similar in shape to the counterion distribution in Fig. 8(a). Figure 9 is a snapshot of the fluid phase containing $N=30$ colloids at packing fraction $\eta=0.11$ and reservoir screening length $\kappa \sigma=5$.

Figures 10(a) and 10(b) show the colloid-colloid, colloid-coion, and colloid-counterion radial distribution functions (RDFs) for two statepoints close to the fluid-bcc melting line. The RDFs in Fig. 10(a) are for packing fraction $\eta=0.206$ and reservoir screening length $\kappa \sigma=2$, and those in Fig. 10(b) are for $\eta=0.266$ and $\kappa \sigma=5$. In Fig. 10, we also plot the RDFs of Yukawa systems, where the screening length $\kappa_{\text {eff }} \sigma$ is from Eq. (6) (the gray dashed and dot-dashed lines) or $\kappa_{\text {eff }}^{\prime} \sigma$ from Eq. (7) (the gray dotted and full lines). We use a constant effective charge $Z_{\text {eff }}=80$ for the RDFs plotted with the dotted and dashed lines, and we use the 


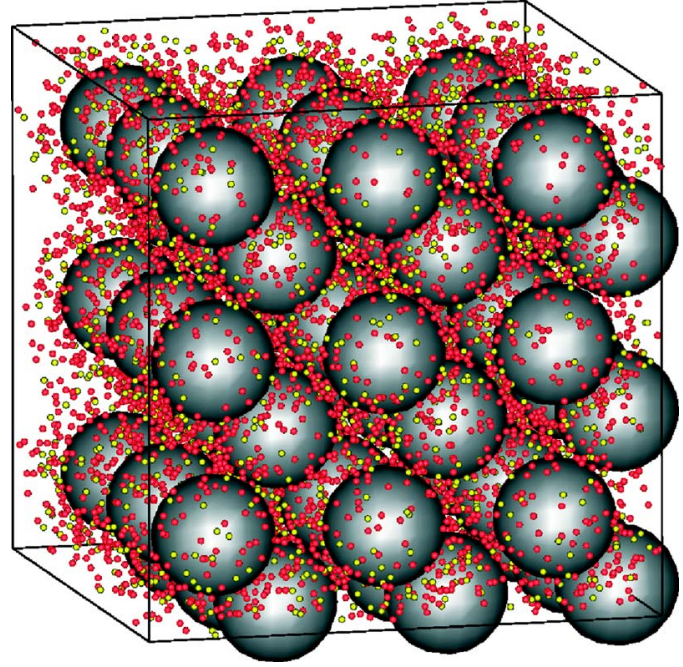

FIG. 7. A snapshot of a bcc crystal with $N=54$ colloids at packing fraction $\eta=0.34$ and reservoir screening length $\kappa \sigma=5$. The small light (yellow) spheres are coions and the small dark (red) spheres are counterions. This system was used to calculate the contour plots in Figs. 8(a)-8(d).

effective charge from the MSA theory, Eq. (8), for the RDFs plotted with the full and dot-dashed lines. We observe that the results with $Z_{\text {eff }}=80$ always underestimate the structure. The best agreement (the gray full lines) is obtained by using $\kappa_{\text {eff }}^{\prime} \sigma$ from Eq. (7) and $Z_{\text {eff }}$ from the MSA theory [Eq. (8)], although the result always slightly overestimates the structure. We want to point out that the primitive model RDFs in Figs. 10(a) and 10(b) can be fitted with $Z_{\text {eff }}=80$ if $\kappa_{\text {eff }} \sigma$ is treated as a fitting parameter. However, from our colloidcolloid RDF data for statepoints in the range $\eta$ $\in[0.03,0.314]$ and $\kappa \sigma \in[0,9.5]$, we noticed that at $\eta \gtrsim 0.2$ the fitting was not possible if $Z_{\text {eff }}=80$ was used. More specifically, at state points $\kappa \sigma=2.0$ and $\eta=0.21, \kappa \sigma=5.0$ and $\eta=0.27, \kappa \sigma=6.9$ and $\eta=0.28, \kappa \sigma=8.7$ and $\eta=0.30$, and

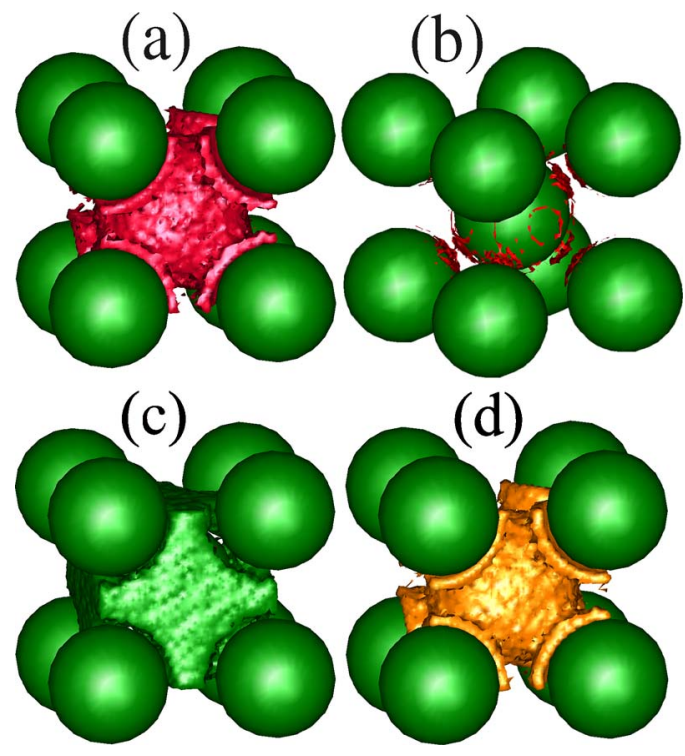

FIG. 8. Constant-density contour plots for [(a) and (b)] counterion, (c) coion, and (d) charge densities in a bcc unit cell at packing fraction $\eta$ $=0.34$ and reservoir screening length $\kappa \sigma=5$. In (a), (c), and (d), the central colloid of the bcc unit cell is covered by the contour plots.

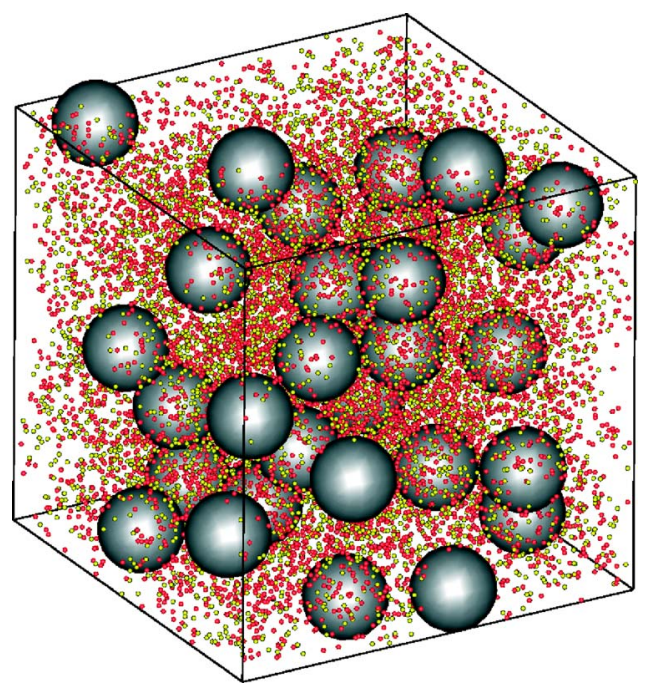

FIG. 9. A snapshot of the fluid phase with $N=30$ colloids at packing fraction $\eta=0.11$ and reservoir screening length $\kappa \sigma=5$. The small light (yellow) spheres are coions and the small dark (red) spheres are counterions.

$\kappa \sigma=9.5$ and $\eta=0.31$, we observed that a higher effective charge was required to make the fitting of the colloid-colloid RDF even qualitative.

In conclusion, our results seem to suggest that, at $\eta$ $>0.2$, the effective screening length is best described by taking into account the excluded volume of the colloids, as we do in Eq. (7), and the effective charge should be chosen larger than the bare charge. At $\eta<0.2$ we find that the colloid-colloid structure can be described by the DLVO theory, i.e., with $Z_{\text {eff }}=80$ and $\kappa_{\text {eff }} \sigma$ from Eq. (6). At these low densities, we also find that the MSA theory gives in certain cases worse description of the structure than the DLVO theory. We emphasize that our results are not meant to validate the MSA theory. We use this theory simply in lack of anything better and acknowledge that it gives only a rough idea of the optimal effective charge.

Note that we have implicitly assumed that the Yukawa form of the pair potential is valid at concentrated colloidal suspensions. This does not need to be so, but we do not have evidence of any other form of the pair potential either.

\section{Melting line}

We determine the melting line for the primitive model at reservoir screening lengths $\kappa \sigma=0.0,2.0,5.0,6.9,8.7$, and 9.5 using the Lindemann criterion (12). The numerical values for the melting points are given in Table I. In Table I, the effective screening lengths $\kappa_{\text {eff }} \sigma$ are calculated using Eq. (6) and $\kappa_{\text {eff }}^{\prime} \sigma$ are calculated using Eq. (7). We also give the corresponding effective charges $Z_{\text {eff }}$ and $Z_{\text {eff }}^{\prime}$ that are calculated using the MSA theory from Eq. (8). We checked that charge renormalization due to condensation is insignificant by calculating $Z_{\text {re }}$ at the statepoints in Table I using the PoissonBoltzmann cell model. ${ }^{25}$ According to our results, the renormalized charge is $Z_{\mathrm{re}} \approx 79$, i.e., very close to the bare charge.

Figure 11 shows the melting line of the primitive model in the $\eta-\kappa_{\text {eff }} \sigma$ representation. The squares mark the results where the mapping onto the $\eta$ - $\kappa_{\text {eff }} \sigma$ plane is done using $\kappa_{\text {eff }} \sigma$ from Eq. (6), and the circles mark the results where the map- 

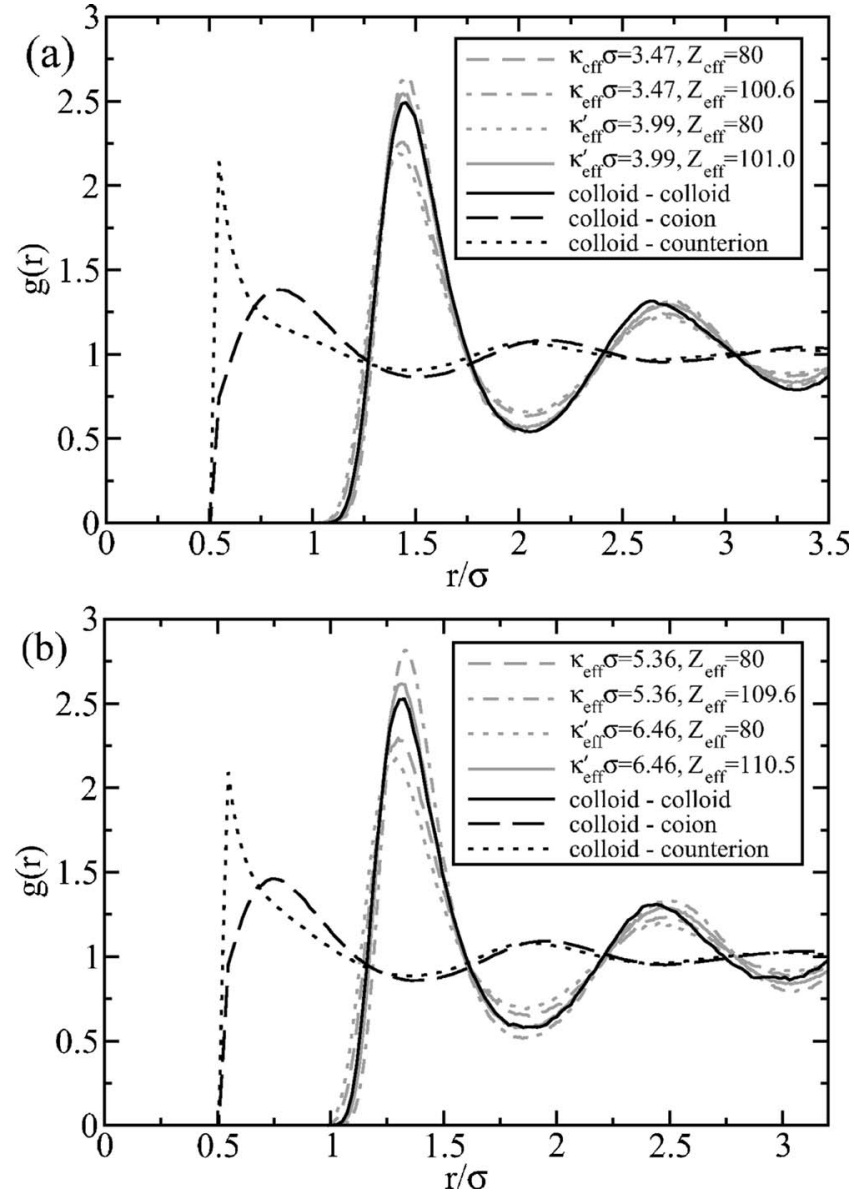

FIG. 10. Radial distribution functions (RDFs) for a system with $N=54$ colloids with charge $Z=80$ close to the fluid-bcc melting line. (a) shows the RDFs for a system at packing fraction $\eta=0.206$ and reservoir screening length $\kappa \sigma=2$. (b) shows the RDFs for a system at packing fraction $\eta$ $=0.266$ and reservoir screening length $\kappa \sigma=5$. The colloid-colloid RDFs are plotted with the full lines, the colloid-coion with the dashed lines, and the colloid-counterion with the dotted lines. The gray lines give the RDFs of Yukawa systems for four different choices of Yukawa parameters, see text.

ping onto the $\eta-\kappa_{\text {eff }} \sigma$ plane is done using $\kappa_{\text {eff }}^{\prime} \sigma$ from Eq. (7). The filled symbols denote the melting points of a bcc crystal, while the open symbols denote the melting points of a fcc crystal. The inset in Fig. 11 shows the melting line in terms of the reservoir screening length $\kappa \sigma$. For comparison, Fig. 11 also shows the phase diagrams of point Yukawa particles (the black dashed and dot-dashed lines) from Ref. 54, which were mapped onto the $\eta-\kappa_{\text {eff }} \sigma$ plane by matching the point

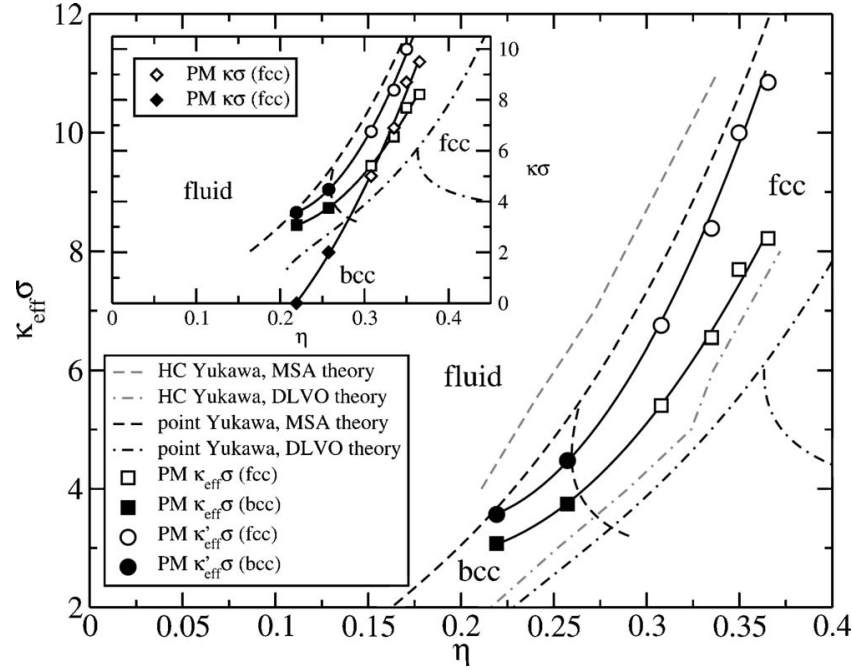

FIG. 11. Phase diagram of charged colloids with charge $Z=80$ and Bjerrum length $\lambda_{B} / \sigma=0.0225$ in the packing fraction $\eta$-effective length $\kappa_{\text {eff }} \sigma$ representation. The point Yukawa phase boundaries from Ref. 54 are denoted by the black dashed and dot-dashed lines and the corresponding hard-core Yukawa melting lines are denoted by the gray lines. The primitive model melting points are denoted by the squares $(\square)$ and circles $(\bigcirc)$. The filled and open symbols are the melting points for bcc and fcc crystals, respectively. The inset shows the melting line [diamonds $(\diamond)]$ in terms of the reservoir screening length $\kappa \sigma$. The full lines are guides to the eye.

Yukawa pair potential with the hard-core pair potential at $r$ $>\sigma$ (for details, see Ref. 4). In the mapping of the dashed lines, we used the effective charge $Z_{\text {eff }}$ from the MSA theory [Eq. (8)], and in the mapping of the dot-dashed lines, we used the DLVO theory bare charge $Z_{\text {eff }}=80$. We also calculated the melting line of hard-core Yukawa particles using the Lindemann criterion. These are plotted by the gray lines, and the effective charges are chosen as in the corresponding point Yukawa phase lines. As can be seen from Fig. 11, the primitive model melting lines are between the Yukawa fluid-solid phase lines, and it is hard to say which mapping of the primitive model and the Yukawa model is the best. Using the DLVO theory, i.e., $\kappa_{\text {eff }} \sigma$ from Eq. (6) and $Z_{\text {eff }}=80$, gives a reasonable agreement between the primitive model results denoted by the squares and the Yukawa fluid-solid line denoted by the dot-dashed line. In this case, the Yukawa model predicts more fluid phase than the primitive model. Using $\kappa_{\text {eff }}^{\prime} \sigma$ from Eq. (7) and $Z_{\text {eff }}$ from the MSA theory [Eq. (8)] gives a reasonable agreement between the primitive model

TABLE I. Numerical values for the primitive model melting line. $\eta_{\text {melt }}$ is the melting packing fraction according to the Lindemann (12), $\kappa_{\mathrm{id}} \sigma$ is the ideal reservoir screening length [see Eq. (4)], $\kappa \sigma$ is the reservoir screening length [see Eq. (2)], $\kappa_{\text {eff }} \sigma$ and $\kappa_{\text {eff }}^{\prime} \sigma$ are the effective screening lengths from Eqs. (6) and (7), respectively, $Z_{\text {eff }}$ and $Z_{\text {eff }}^{\prime}$ are the corresponding effective charges from the MSA theory [Eq. (8)], and $\left\langle\rho_{S}\right\rangle \sigma^{3}$ is the average salt density.

\begin{tabular}{|c|c|c|c|c|c|c|c|c|}
\hline Crystal & $\eta_{\text {melt }}$ & $\kappa_{\mathrm{id}} \sigma$ & $\kappa \sigma$ & $\kappa_{\mathrm{eff}} \sigma$ & $Z_{\text {eff }}$ & $\kappa_{\mathrm{eff}}^{\prime} \sigma$ & $Z_{\text {eff }}^{\prime}$ & $\left\langle\rho_{S}\right\rangle \sigma^{3}$ \\
\hline bcc & 0.219 & 0 & 0.0 & 3.08 & 101.9 & 3.57 & 102.3 & 0.0 \\
\hline bcc & 0.257 & 2 & 2.0 & 3.74 & 107.1 & 4.48 & 107.7 & 5.1 \\
\hline fcc & 0.284 & 5 & 5.0 & 5.39 & 112.0 & 6.75 & 116.7 & 29.8 \\
\hline fcc & 0.335 & 7 & 6.9 & 6.55 & 120.6 & 8.39 & 122.4 & 50.2 \\
\hline fcc & 0.350 & 9 & 8.7 & 7.69 & 124.1 & 10.00 & 126.3 & 77.9 \\
\hline fcc & 0.366 & 10 & 9.5 & 8.22 & 129.3 & 10.85 & 129.8 & 91.5 \\
\hline
\end{tabular}


results denoted by the circles and the Yukawa fluid-solid line denoted by the dashed line. In this case, the Yukawa model predicts less fluid phase than the primitive model. As noted in Sec. III B, at $\eta>0.2$ the agreement of the colloid-colloid RDFs of the primitive model and the Yukawa system is the best in this case. We have also calculated the melting point for a system with $Z=160$ and no added salt $(\kappa \sigma=0)$. We found $\eta_{\text {melt }}=0.066 \pm 0.005$ for a bcc crystal with $N=54$ colloids. At this density, $\kappa_{\mathrm{eff}} \sigma=2.47$ and $\kappa_{\mathrm{eff}}^{\prime} \sigma=2.58$ from Eqs. (6) and (7), respectively. Using $\kappa_{\text {eff }} \sigma=2.5$ and $Z_{\text {eff }}=160$ in the hard-core Yukawa model gives melting at $\eta_{\text {melt }}=0.071$, in good agreement with the primitive model result. This shows that at low densities, the DLVO theory gives a good description of charged colloids (as long as charge renormalization is not important). We can only conclude that it is not clear how the mapping between the primitive model and the Yukawa model should be done in the packing fraction range $\eta>0.2$.

In our earlier work, ${ }^{23,24}$ we performed simulations of the effective one-component system using a repulsive two-body Yukawa potential and an attractive three-body potential, and we found broad fluid-fcc and bcc-fcc coexistence regions at $\kappa \sigma=2.2-2.45$. Using this effective three-body potential approach, the simulations showed a clear phase separation for statepoints well inside such a broad two-phase coexistence region. This is in contrast with our primitive model simulations, which do not give any indication of phase separation or broad coexistence regions when the same parameters are used as in Refs. 23 and 24.

\section{CONCLUSIONS}

We develop an efficient simulation method to study suspensions of charged spherical colloids using the primitive model. The combination of the fine lattice discretization approach and cluster moves enables us to speed up sampling. We find the cluster moves essential to make the primitive model simulations feasible in the colloidal regime. These methods are a combination of methods used previously. ${ }^{34,43-46}$ We showed that by increasing the lattice discretization, its effect on the results can be made small in a sense that no change is observed if the lattice is refined further. The crucial difference between our work and the earlier work is that we perform the simulations in the semigrand canonical ensemble, where the volume, temperature, number of colloids, and salt chemical potential are fixed. This allows us to make direct comparison not only with experiments but also with various theoretical approaches. For instance, Refs. 55 and 56 show direct comparisons of radial distribution functions measured experimentally using confocal microscopy and those obtained from our primitive model code.

We studied charge distributions in a bec crystal by plotting contour plots of coion, counterion, and charge (counterion minus coion) densities. The charge distributions were found to be in qualitative agreement with the DLVO theory, which predicts spherical double layers around each colloid.

We determined the fluid-solid melting line of the primitive model for colloidal charge $Z=80$ using the Lindemann criterion and compared it with the melting line of colloids described by the effective Yukawa potential of the DLVO theory. We found that the agreement between the primitive model and the Yukawa model depends strongly on the way the mapping between the two systems is performed, i.e., on the choice of the effective screening length and effective charge. Our results for colloid-colloid RDFs at $\eta>0.2$ suggest that the effective screening length is best described by taking into account the excluded volume of the colloids and that the effective charge is higher than the bare charge. This charge renormalization is due to the excluded volume of the colloids that reduces the volume available for the microions and therefore reduces the screening. ${ }^{30,51}$ Thus, the colloids feel a stronger repulsion because their charges are less screened. However, we do not have direct evidence confirming this mechanism. At $\eta<0.2$, the colloid-colloid structure can be described by using an effective charge equal to the bare charge, i.e., $Z_{\text {eff }}=80$. We are not aware of a unique way of mapping between the primitive model and the Yukawa model that would work at all packing fractions, except fitting. ${ }^{30}$ One possibility is that the Yukawa form of the pair potential breaks down at high densities, but our results did not show any definite evidence supporting this. Note that the breakdown of the Yukawa form of the pair potential has been observed before by other authors. ${ }^{15,16,49}$

Treating the salt grand canonically allows us to make a direct comparison with our earlier work, ${ }^{23,24}$ where threebody attraction gave rise to broad fluid-fcc and bcc-fcc coexistence regions. We use the same parameters as in Refs. 23 and 24, but do not find any broad coexistence regions or any other manifestations of many-body interactions. This suggests that the four- and higher-body terms play an essential role in the parameter range considered here; they seem to cancel the effective three-body attraction. Therefore, the effective colloid interactions in colloidal suspensions seem to be better described by an effective pair potential description than by an effective Hamiltonian truncated after the threebody term, as was done in Refs. 23 and 24.

\section{ACKNOWLEDGMENTS}

The authors thank A. Z. Panagiotopoulos for providing the original lattice Monte Carlo code and for careful reading of the manuscript. The authors thank B. Zoetekouw for providing the Poisson-Boltzmann cell model solver, and $\mathrm{R}$. van Roij, P. Linse, and A. R. Denton for useful discussions and for careful reading of the manuscript. This work is part of the Research program of the "Stichting voor Fundamenteel Onderzoek der Materie (FOM)," which is financially supported by the "Nederlandse Organisatie voor Wetenschappelijk Onderzoek (NWO)."

${ }^{1}$ B. Derjaguin and L. Landau, Acta Physicochim. URSS 14, 633 (1941).

${ }^{2}$ E. J. W. Verwey and J. Th. G. Overbeek, Theory of the Stability of Lyotropic Colloids (Elsevier, Amsterdam, 1948).

${ }^{3}$ M. O. Robbins, K. Kremer, and G. S. Grest, J. Chem. Phys. 88, 3286 (1988).

${ }^{4}$ A.-P. Hynninen and M. Dijkstra, Phys. Rev. E 68, 021407 (2003).

${ }^{5}$ Y. Monovoukas and A. P. Gast, J. Colloid Interface Sci. 128, 533 (1989).

${ }^{6}$ E. B. Sirota, H. D. Ou-Yang, S. K. Sinha, P. M. Chaikin, J. D. Axe, and Y. Fujii, Phys. Rev. Lett. 62, 1524 (1989).

${ }^{7}$ H. J. Schöpe, T. Decker, and T. Palberg, J. Chem. Phys. 109, 10068 (1998).

${ }^{8}$ A. Yethiraj and A. van Blaaderen, Nature (London) 421, 513 (2003). 
${ }^{9}$ B. V. R. Tata, M. Rajalakshmi, and A. K. Arora, Phys. Rev. Lett. 69, 3778 (1992); T. Palberg and M. Wurth, ibid. 72, 786 (1994).

${ }^{10}$ K. Ito, H. Yoshida, and N. Ise, Science 263, 66 (1994).

${ }^{11}$ B. V. R. Tata, E. Yamahara, P. V. Rajamani, and N. Ise, Phys. Rev. Lett. 78, 2660 (1997).

${ }^{12}$ A. E. Larsen and D. G. Grier, Nature (London) 385, 230 (1997).

${ }^{13}$ C. Russ, H. H. von Grünberg, M. Dijkstra, and R. van Roij, Phys. Rev. E 66, 011402 (2002).

${ }^{14}$ Note that although the effective three-body interactions are attractive, the total force is still repulsive.

${ }^{15}$ M. Brunner, C. Bechinger, W. Strepp, V. Lobaskin, and H. H. von Grünberg, Europhys. Lett. 58, 926 (2002).

${ }^{16}$ R. Klein, H. H. von Grünberg, C. Bechinger, M. Brunner, and V. Lobaskin, J. Phys.: Condens. Matter 14, 7631 (2002).

${ }^{17}$ M. Brunner, J. Dobnikar, and H. H. von Grünberg, Phys. Rev. Lett. 92, 078301 (2004).

${ }^{18}$ C. Russ, M. Brunner, C. Bechinger, and H. H. von Grünberg, Europhys. Lett. 69, 468 (2005).

${ }^{19}$ J. Dobnikar, M. Brunner, J. Baumgartl, C. Bechinger, and H. H. von Grünberg, Proc. SPIE 5514, 340 (2004).

${ }^{20}$ J. Dobnikar, Y. Chen, R. Rzehak, and H. H. von Grünberg, J. Chem. Phys. 119, 4971 (2003).

${ }^{21}$ J. Dobnikar, R. Rzehak, and H. H. von Grünberg, Europhys. Lett. 61, 695 (2003).

${ }^{22}$ J. Dobnikar, Y. Chen, R. Rzehak, and H. H. von Grünberg, J. Phys.: Condens. Matter 15, S263 (2003).

${ }^{23}$ A.-P. Hynninen, M. Dijkstra, and R. van Roij, J. Phys.: Condens. Matter 15, S3549 (2003).

${ }^{24}$ A.-P. Hynninen, M. Dijkstra, and R. van Roij, Phys. Rev. E 69, 061407 (2004).

${ }^{25}$ S. Alexander, P. M. Chaikin, P. Grant, G. J. Morales, P. Pincus, and D. Hone, J. Chem. Phys. 80, 5776 (1984).

${ }^{26}$ F. Bitzer, T. Palberg, H. Löwen, R. Simon, and P. Leiderer, Phys. Rev. E 50, 2821 (1994).

${ }^{27}$ L. Belloni, Colloids Surf., A 140, 227 (1998).

${ }^{28}$ H. Löwen, P. A. Madden, and J.-P. Hansen, Phys. Rev. Lett. 68, 1081 (1992).

${ }^{29}$ H. Löwen, J.-P. Hansen, and P. A. Madden, J. Chem. Phys. 98, 3275 (1993).

${ }^{30}$ H. Löwen and G. Kramposthuber, Europhys. Lett. 23, 673 (1993).
${ }^{31}$ R. Car and M. Parrinello, Phys. Rev. Lett. 55, 2471 (1985).

${ }^{32}$ D. Frenkel and B. Smit, Understanding Molecular Simulations, 2nd ed. (Academic, New York, 2002).

${ }^{33}$ P. P. Ewald, Ann. Phys. 64, 253 (1921).

${ }^{34}$ A. Z. Panagiotopoulos and S. K. Kumar, Phys. Rev. Lett. 83, 2981 (1999).

${ }^{35}$ J. M. Romero-Enrique, G. Orkoulas, A. Z. Panagiotopoulos, and M. E. Fisher, Phys. Rev. Lett. 85, 4558 (2000).

${ }^{36}$ A. Z. Panagiotopoulos and M. E. Fisher, Phys. Rev. Lett. 88, 045701 (2002).

${ }^{37}$ D. Cheong and A. Z. Panagiotopoulos, J. Chem. Phys. 119, 8526 (2003).

${ }^{38}$ A.-P. Hynninen, M. Dijkstra, and A. Z. Panagiotopoulos, J. Chem. Phys. 123084903 (2005)

${ }^{39}$ G. Orkoulas, S. K. Kumar, and A. Z. Panagiotopoulos, Phys. Rev. Lett. 90, 048303 (2003)

${ }^{40}$ A. Z. Panagiotopoulos, J. Chem. Phys. 123104504 (2005).

${ }^{41} \tau$ is defined as the time it takes to calculate the total potential energy ten times on a Pentium $43 \mathrm{GHz} \mathrm{CPU}$.

${ }^{42}$ J. W. Perram, H. G. Petersen, and S. W. de Leeuw, Mol. Phys. 65, 875 (1988).

${ }^{43}$ V. Lobaskin and K. Qamhieh, J. Phys. Chem. B 107, 8022 (2003).

${ }^{44}$ V. Lobaskin and P. Linse, J. Chem. Phys. 1114300 (1999).

${ }^{45}$ P. Linse and V. Lobaskin, Phys. Rev. Lett. 834208 (1999).

${ }^{46}$ P. Linse, Adv. Polym. Sci. 185, 111 (2005).

${ }^{47}$ A. R. Denton, Phys. Rev. E 62, 3855 (2000).

${ }^{48}$ T. Palberg, W. Mönch, F. Bitzer, R. Piazza, and T. Bellini, Phys. Rev. Lett. 74, 4555 (1995).

${ }^{49}$ V. Lobaskin, M. Brunner, C. Bechinger, and H. H. von Grünberg, J. Phys.: Condens. Matter 15, 6693 (2003).

${ }^{50}$ A.-P. Hynninen and M. Dijkstra, J. Phys.: Condens. Matter 15, S3557 (2003).

${ }^{51}$ L. Belloni, J. Chem. Phys. 85, 519 (1986).

${ }^{52}$ F. A. Lindemann, Z. Phys. 11, 609 (1910).

${ }^{53}$ http://www.csc.fi/gopenmol/

${ }^{54}$ S. Hamaguchi, R. T. Farouki, and D. H. E. Dubin, Phys. Rev. E 56, 4671 (1997).

${ }^{55}$ C. P. Royall, R. van Roij, and A. van Blaaderen, J. Phys.: Condens. Matter 17, 2315 (2005).

${ }^{56}$ C. P. Royall, M. E. Leunissen, A.-P. Hynninen, M. Dijkstra, and A. van Blaaderen (submitted). 